\title{
Millisecond pulsar observation system using AOS
}

\author{
Y. Hanado, M. Imae, and M. Sekido \\ Communications Research Laboratory (CRL), Japan
}

\section{Introduction}

Communications Research Laboratory (CRL) developed a new millisecond pulsar observation system which used an Acousto-Optic Spectrometer (AOS)[1]. An AOS is simple and effective at dividing a wide band signal into many channels simultaneously[2]. Using this system, we started preliminary observations of PSR1937+21 for the purpose of precise measurements of millisecond pulsar's pulse timing.

\section{Observation system}

Fig. 1 shows a block diagram of our observation system using the $34 \mathrm{~m}$ antenna of CRL. This antenna has several receivers $(1.5,2,5,8,10,15,22,43 \mathrm{GHz})$ with selective polarizer. We use $1.5 \mathrm{GHz}$ or $2 \mathrm{GHz}$ band for pulsar observations. An IF signal with $50 \mathrm{MHz}$ bandwidth is divided to $200 \mathrm{kHz} \times 256 \mathrm{ch}$ by the AOS. The speed of data transporting rate from the camera is about $13 \mu \mathrm{s}$ which limits a time resolution. We get 100 bins per one period for PSR $1937+21$. The video averaging processor works as two 8 bits $A / D$ converters and an averager which allows $2^{24}$ pulses' addition in each channel. The averaged data of each channel are combined after de-dispersion processing which is carried out in $1 / 1000$ steps of the pulsar period. ¿From this final profile, the peak phase is defined as arrival pulse timing. Host\#2 calculates the a priori pulse period by TEMPO program, and supplies it in real time to the synthesizer which controls the averaging trigger clock of the timing signal generator. The reference clock of this system is synchronized with UTC (CRL) via the GPS satellites. The difference between the reference clock and the internal clock of the timing signal generator is monitored using a time interval counter.

\section{Observation of PSR1937+21}

Preliminary observations of PSR1937+21 were carried out on 6 days during 2 months in 1995 . The observation frequency was $2 \mathrm{GHz}$. One pulse profile was made after averaging 524288 pulses (= integrating about 15 minutes), and 6-8 sets were obtained in one day. From these averaged profile the peak phases were defined, and their residuals from the a priori value show a pulse timing stability. Residual, $R(t)$ is calculated as below:

$$
R(t)=\phi_{o b s}(t)-\phi_{c a l c}(t),
$$


where $\phi_{o b s}(t)$ is a observed peak phase, and $\phi_{c a l c}(t)$ is an a priori phase calculated by TEMPO.

Fig. 2 plots the phase fluctuation in one day, $R^{\prime}(t)$ :

$$
R^{\prime}(t)=R(t)-R_{\text {ave }},
$$

where $R_{\text {ave }}$ is the average of $R(t)$ values in each day. The standard deviation of $R^{\prime}(t)$ in all data is about $7.1 \mu \mathrm{s}$, which is approximately same as the time resolution. This rather large value is due to define a peak phase from one point of the peak. A fitting method for the peak phase definition will make such a fluctuation smaller.

\section{Conclusion}

The performance of our new system was confirmed by preliminary observations of PSR $1937+21$, and the results was reasonable in terms of the $34 \mathrm{~m}$ antenna's specifications. Now some improvements are progressing. We will expand the current system to 4 units for $200 \mathrm{MHz}$ bandwidth which will be a effective tool for precise pulsar-timing study.

\section{References}

[1] Y. Hanado, M. Imae and M. Sekido, "Millisecond pulsar Observation System Using Acousto-Optic Spectrometer," IEEE Trans. vol.44, No.2, pp.107109, April, 1995.

[2] A. P. Goutzoulis and I. J. Abramovitz, "Digital electronics meets its match, "IEEE Spectrum, pp.21-25, August 1988.

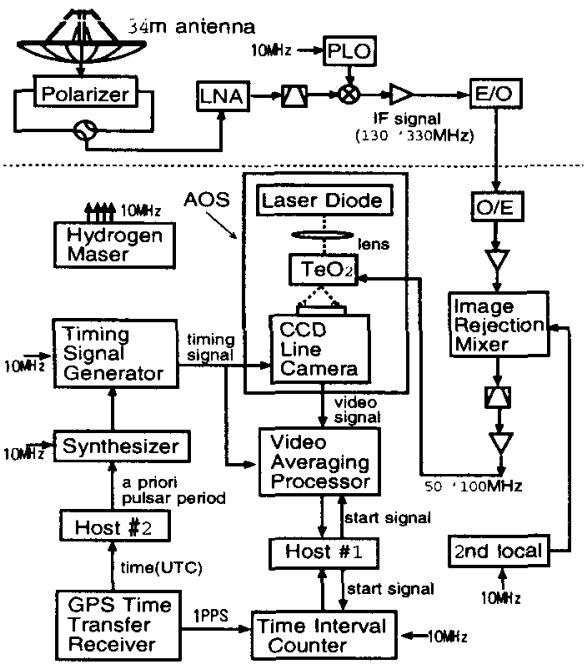

Figure 1.

Block diagram of the observation system at CRL.

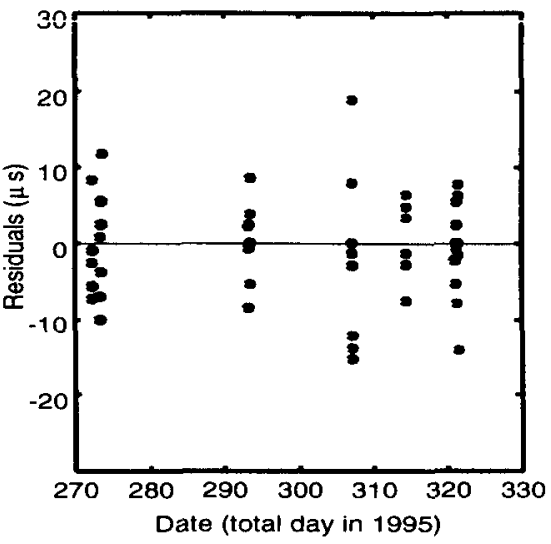

Figure 2.

Residuals of observed pulse timing from calculated one. The observed phases different days were not continuous, so the each day's average is subtracted from each phase. The observed phases in the same day were continuous. 